\title{
Climate variability and density-dependent population dynamics: Lessons from a simple high-Arctic ecosystem
}

\author{
Dominique Fauteux ${ }^{1}$, Audun Stien ${ }^{2}$, Nigel Yoccoz ${ }^{2}$, Eva Fuglei ${ }^{3}$, and Rolf Ims $^{2}$ \\ ${ }^{1}$ Canadian Museum of Nature \\ ${ }^{2}$ University of Troms $\varnothing$ \\ ${ }^{3}$ Norwegian Polar Institute
}

April 7, 2021

\begin{abstract}
Ecologists are still puzzled by the diverse population dynamics of herbivorous small mammals that range from high-amplitude, multi-annual cycles to stable dynamics. Theory predicts that this diversity results from combinations of climatic seasonality, weather stochasticity and density-dependent food web interactions. The almost ubiquitous 3-5-yr cycles in boreal and arctic climates may theoretically result from bottom-up (plant-herbivore) and top-down (predator-prey) interactions. Assessing empirically the roles of such interactions, and how they are influenced by environmental stochasticity, has been hampered by food web complexity. Here, we take advantage of a uniquely simple High-Arctic food web, which allowed us to analyze dynamics of a graminivorous vole population not subjected to top-down regulation. This population exhibited high-amplitude, non-cyclic fluctuations - partly driven by weather stochasticity. However, the predominant driver of the dynamics was direct density dependence, which alternated between being weak in summer and strong (overcompensatory) in winter that the population frequently crashed. Model simulations showed that this season-specific density dependence would yield regular 2-year cycles in absence of stochasticity. While such short cycles have not yet been observed in mammals, they are theoretically plausible if graminivorous vole populations are deterministically bottom-up regulated. When incorporating weather stochasticity in the model simulations, cyclicity became disrupted and the amplitude was increased - akin to the observed dynamics. Our findings contrast with the 3-5-yr population cycles involving delayed density dependence that are typical of graminivorous small mammals in more complex food webs, suggesting that top-down regulation is an important component of such dynamics.
\end{abstract}

\section{Hosted file}

MS_SiblingvolesDemography_final.pdf available at https://authorea.com/users/393018/articles/ 506750-climate-variability-and-density-dependent-population-dynamics-lessons-from-asimple-high-arctic-ecosystem 